\title{
Statistics and Mean Filters Based on Spatial Noise: A Comparative Study
}

\author{
Ali Abdulkarem Habib \\ Assistant L, Faculty of Computer Science and Mathematics, University of Kufa, Najaf, Iraq
}

\begin{abstract}
The main idea of this paper to exhibit study which illuminate set of characteristics among statistics and mean filters rely on additive and multiplicative in addition salt and pepper noise with compare among them using difference type analysis methods and blur metric. We selected one filter form statistics filters and another one from means filters. Finally, we get perfect results tell as what is the best filter and affect them on noises [3].
\end{abstract}

Keywords: Default color image, statistics and means filters, noise models, blur metric.

\section{INTRODUCTION}

The noise is random variation of brightness or color There is different type of noise model like Gaussian which information in images, and is usually an aspect of most common model, Rayleigh, Erlang, Exponential, electronic. It can produced by the sensor and circuitry of Uniform and Impulse (Salt and pepper).

scanner or digital camera. Image noise can also originate in grain and in the unavoidable shot noise of an ideal In this paper we work on two type of Gaussian noise photon detector. Image noise is an undesirable by-product (additive and multiplicative ) and Uniform in addition to of image capture that adds spurious and extraneous Impulse all these type share in two type filter to remove information.
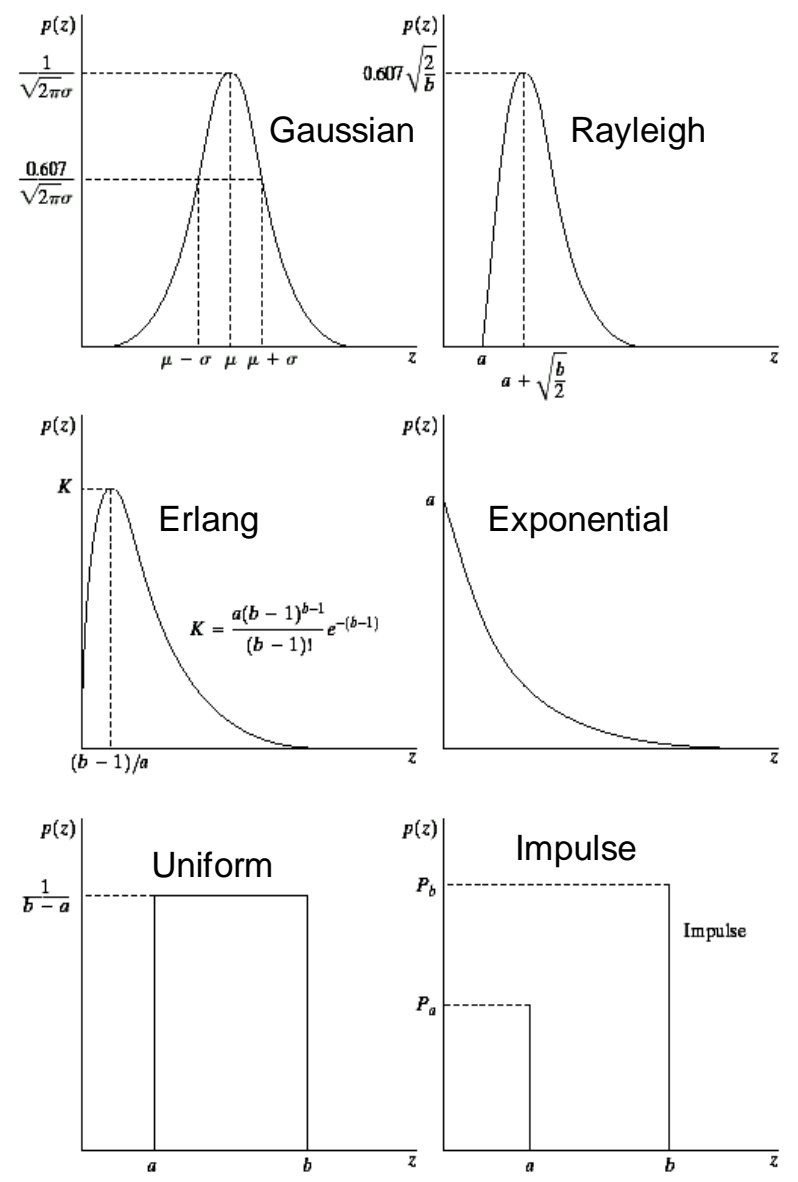

Fig.1. noise models Impulse all these type share in two type filter to remove
them one from means filters called as Contra Harmonic mean and the another one from statistics filters which have many type and we select alpha trimmed. Figure (1) show different type of noise models.

Most masks apply on image using matlab tools where in this survey framing degradation process by math models, which make work on them, became flexible for changeability according to characteristic of damaged image. In addition, we focus on colored image to stand out blur effect to be able to analyze image optimally to know the amount of loss data through standard measures and the degree of smoothing developments in the processed image

\section{ADD NOISE TO IMAGE}

As we mentioned earlier the noise can be used to any matrix (1D, 2D, 3D, n D) where additive noise sums a random value to the image value at each pixel and multiplicative noise replaces a pixel value with a random value with reference to Salt and Pepper noise replaces a pixel value with the maximum or minimum possible values.

For additive Gaussian to use three types of parameters in addition to original image noise name, disparity and scope where second parameter is the dissimilarity of the Gaussian noise added, moreover the last parameter means the perceptual of pixels affected by the noise.

On the other hand multiplicative Gaussian noise need to three arguments image name, noise name and scope.

That type of noise require third parameter value on the contrary, in the first one, it is optional. Figure (2) show effect additive and multiplicative Gaussian noise on image 
Vol. 5, Issue 8, August 2016

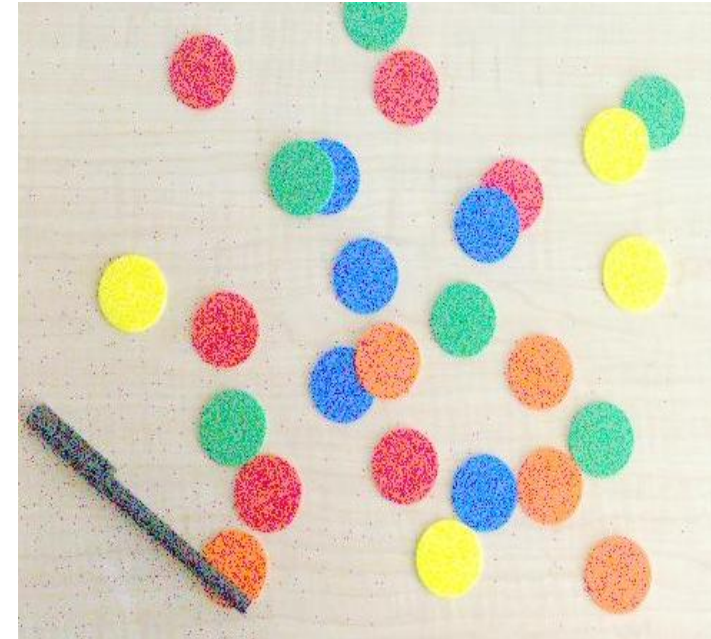

Fig. $(2-$ A) Additive Gaussian noise

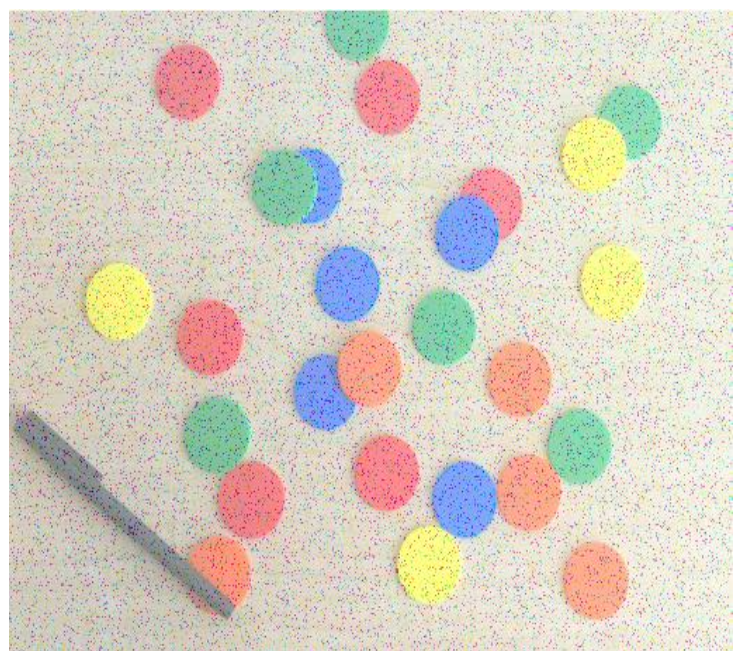

Fig. (2- B) Multiplicative Gaussian

When need to apply uniform noise we found it have same parameter of multiplicative Gaussian noise except disparity always set to maximum image value.

Figure (3) show characteristic of uniform noise.

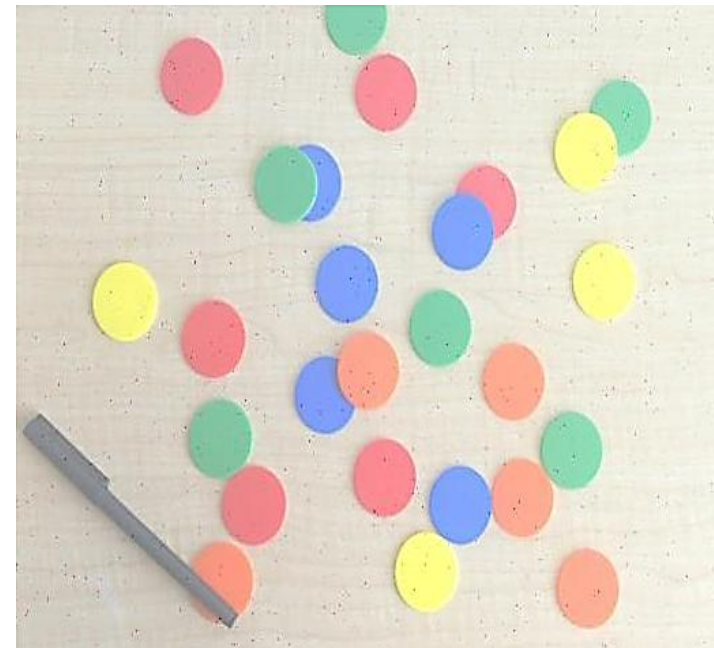

Fig.3 Uniform noise

Also in this paper, we try to apply pepper and salt noise individually to study the characteristics of each one separately, first of all will generating a random matrix of size $m \times n$ which is the size of image, whose value of each element is randomly distributed over a range of 0 to 255 .

When setting that pixel value ' 0 ' whose pixel value is below or equal ' 4 ' this step will add the pepper noise in the image will add the pepper noise in the image, as for the salt noise put pixel value to 255 whose pixel value is $>=$ 251. Figure (4) show how to add salt and pepper noise model to image.

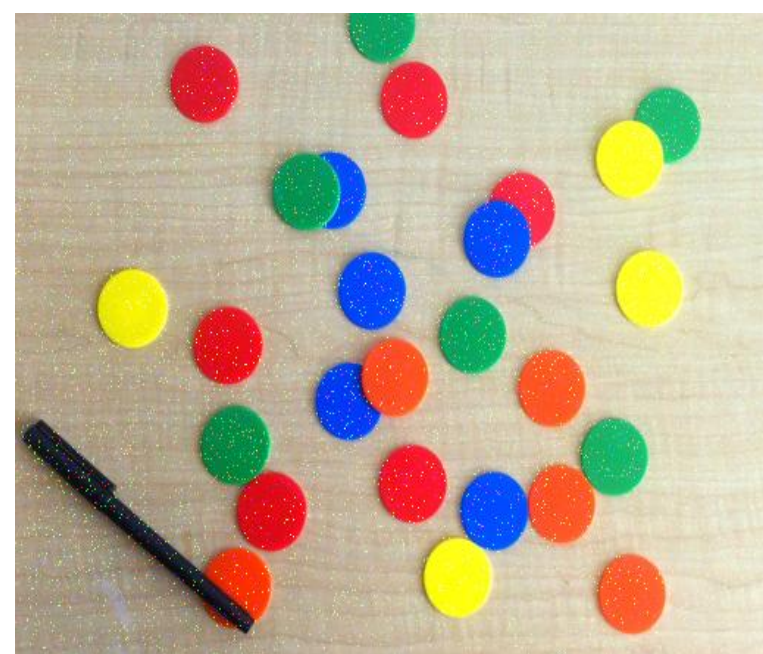

Fig. (4 - A) Salt noise

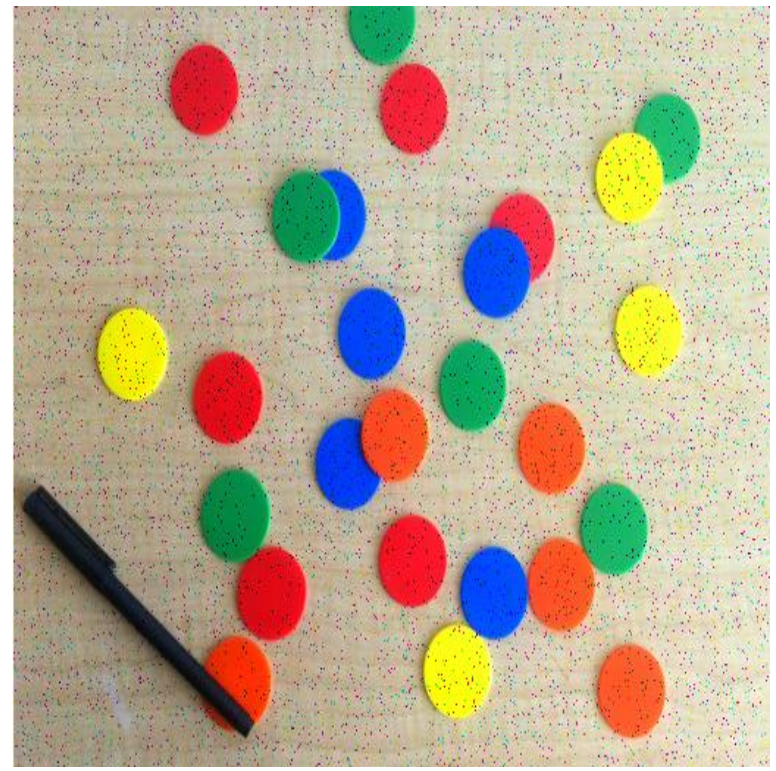

Fig. (4 - B) Pepper noise

\section{REMOVAL NOISE}

We can remove noise from degraded image in spatial or frequency domain. In this paper, we decided to process image in first domain based on selected filters, which need to compare with them. Figure (5) show block diagram of the process of adding and removing noise [3]. 


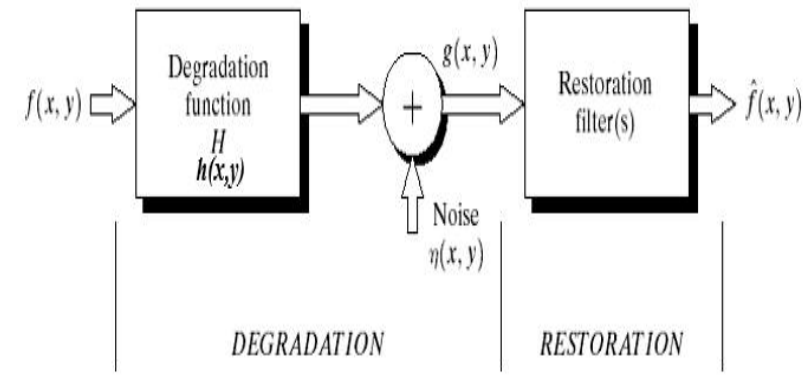

Figure (5) degradation and restoration process

\section{A. Alpha Trimmed Mean Filter}

Its type of order statistics filters refers to statistical methods that depend only on the ordering of the data and not on its numerical values, where average of the data, while easy to compute and very important as an estimate of a central value, is not an order statistic [5].

Median the most commonly used order statistic, the value in the middle position in the sorted order of the values [1].

To remove noise from image using alpha trimmed filter then we have to set $S_{x y}$ be a neighborhood of size $M \times N$, of a point $(\mathrm{x}, \mathrm{y})$ in the given image and let number $\mathrm{d}$, which represent delete $d / 2$ largest and $d / 2$ smallest gray-level values in $S_{x y}$. So denote the remaining gray-level values as $\mathrm{I}_{\mathrm{d}}$.

Finally, we have to take the arithmetic mean of rest of the gray-level values. As eq. (1).

$$
\hat{f}(x, y)=\frac{1}{M N-d} \sum_{(s, t) \in S_{x u}} I_{d}(s, t)
$$

Figures (6) from (A - E) show effect filter on degraded image for all types of noise.

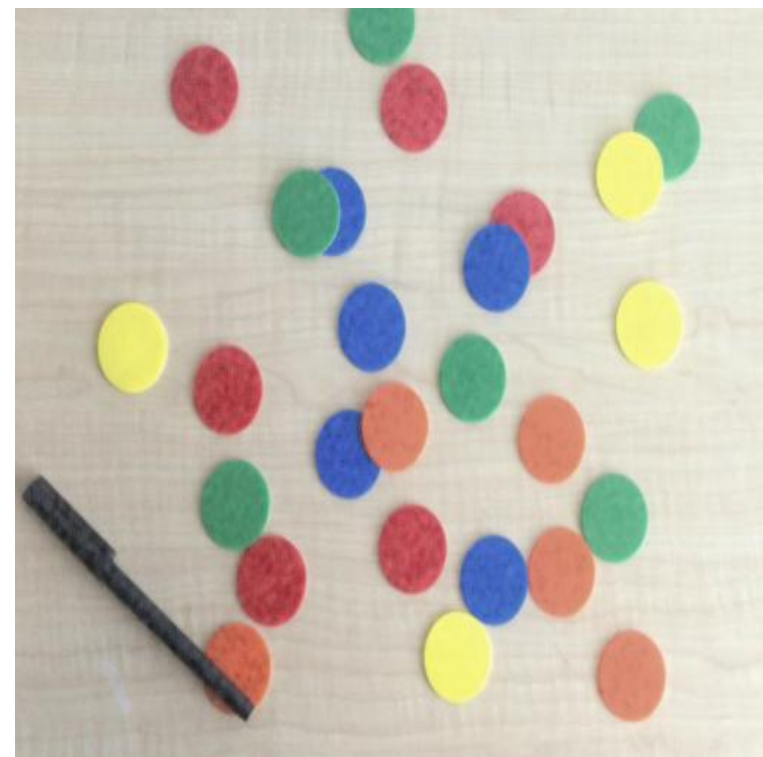

Fig. (6 - A) remove additive Gaussian noise

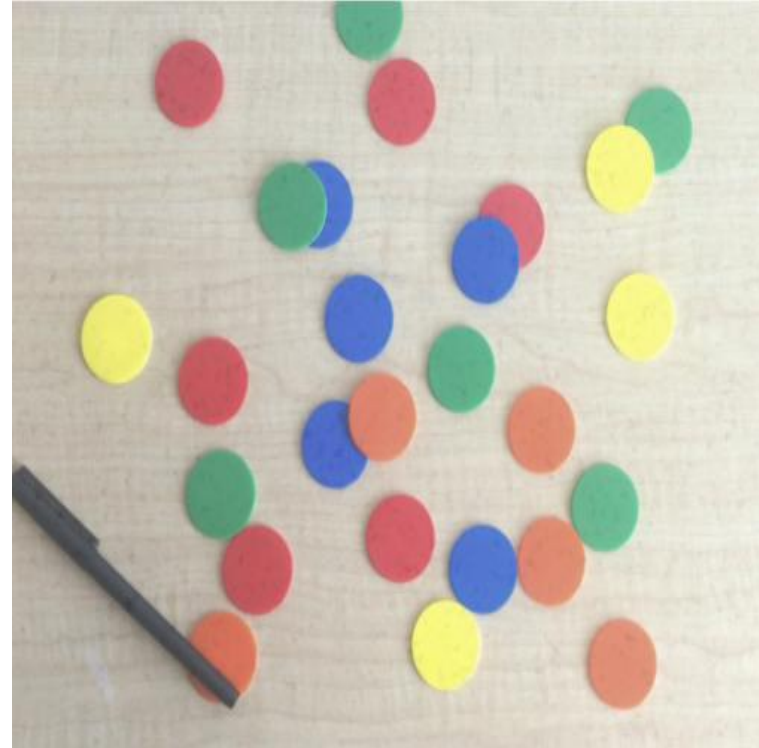

Fig. (6 - B) remove multiplicative Gaussian noise

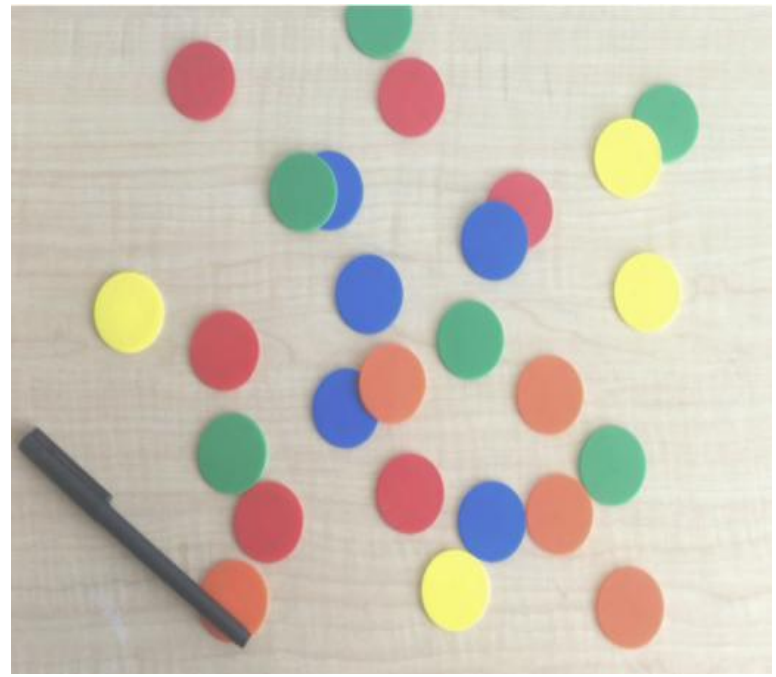

Fig. $(6-C)$ remove uniform noise

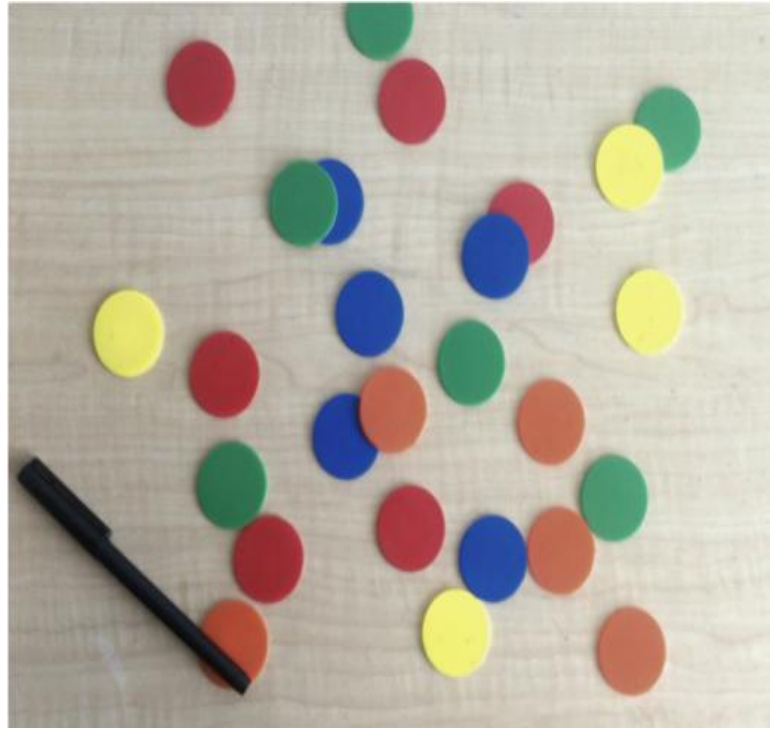

Fig. (6 - D) remove pepper noise 
Vol. 5, Issue 8, August 2016

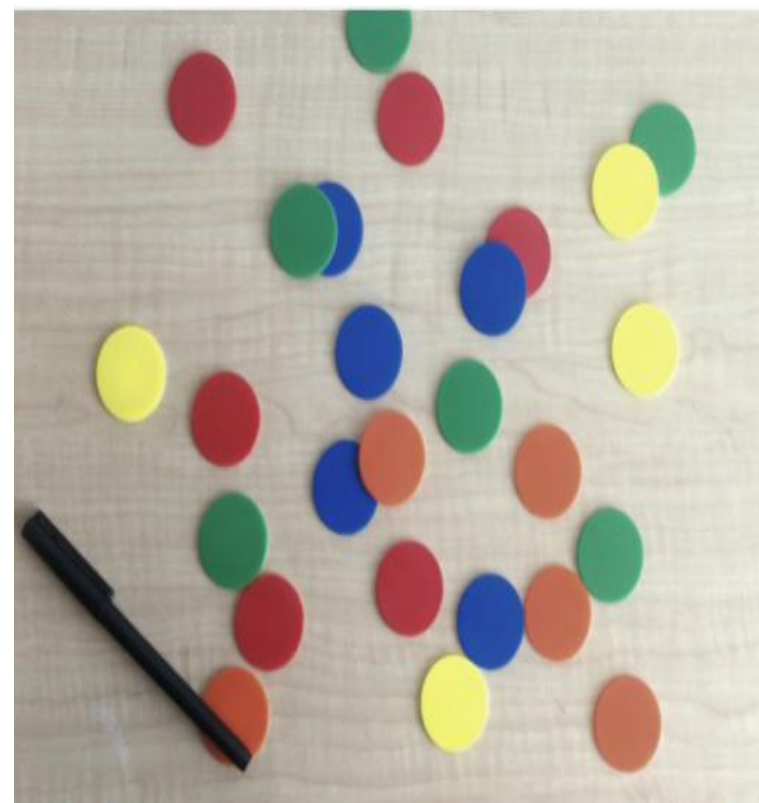

Figure $(6-\mathrm{e})$ remove salt noise

\section{B. Contra harmonic Mean Filter}

This filter the best one from type mean which which exhibit different behaviour because it focus on salt and pepper separately based on Q parameter, whenever the Q is negative value then the mask will eliminates salt noise, whereas positive value of $\mathrm{Q}$, it eliminates pepper noise[3]. As eq. (2),

$$
\hat{f}(x, y)=\frac{\sum_{(s, t) \in S_{x y}} g(s, t)^{Q+1}}{\sum_{(s, t) \in S_{x y}} g(s, t)^{Q}}
$$

Figure $(7-a)$ and figure $(7-b)$ show work of filter.

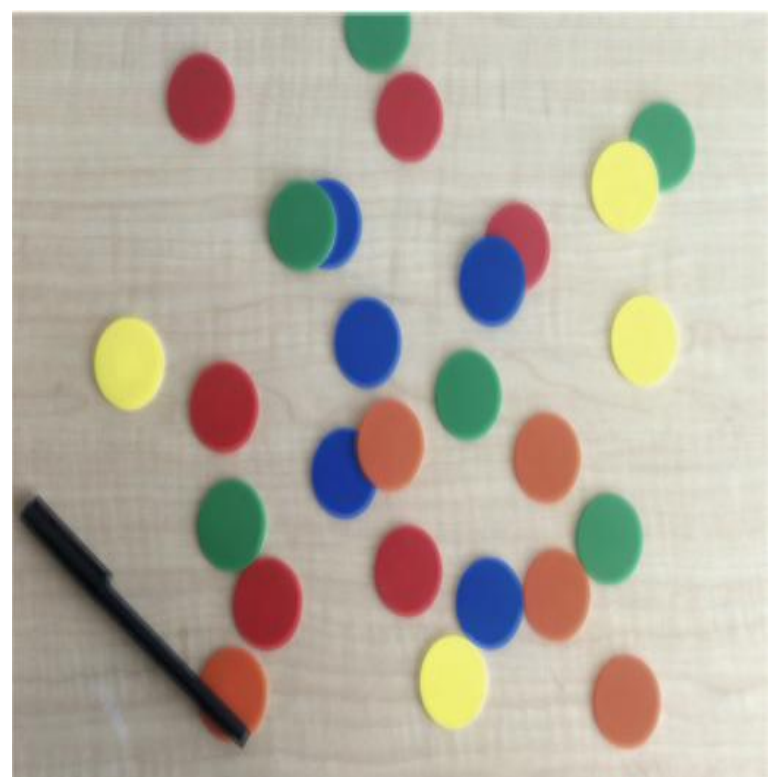

Fig. $(7-\mathrm{A})$ remove pepper noise with $+\mathrm{Q}$

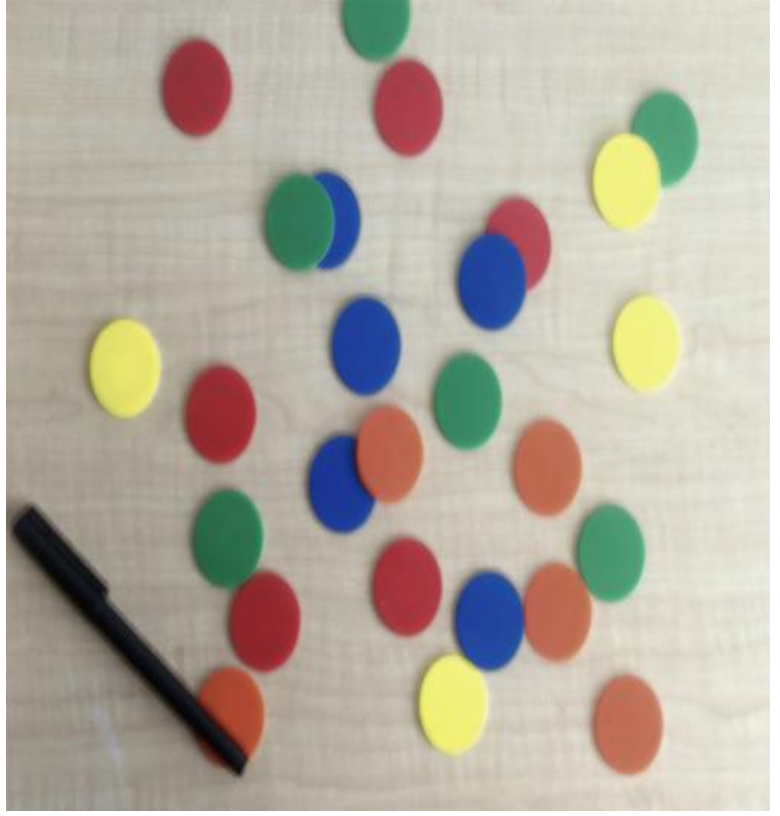

Fig. (7 - B) remove salt noise with -Q

\section{RESULTS AND ANALYSIS}

The main idea from this paper to compare among selected filters, where we used two types of analysis, the first one is standard measurement MSE and PSNR, the first tool demonstrates a difference between the distorted images and processed image whenever MSE value larger than zero then both image not same, as show in table(1).

$$
M S E=\frac{1}{m n} \sum_{i=0}^{m-1} \sum_{j=0}^{n-1}[I(i, j)-K(i, j)]^{2}
$$

Table (1) to show MSE after process image using alpha trimmed mean filter.

\begin{tabular}{|l||l||l|}
\hline Image A & Image B & MSE \\
\hline $\mathrm{Mg}$ & AlphaMg & 2.8211 \\
\hline $\mathrm{Mu}$ & AlphaMu & 0.5338 \\
\hline pepper & Alpha_pepper & 6.5763 \\
\hline Saltim & alphaSaltIM & 6.4818 \\
\hline $\mathrm{Ag}$ & Alpha_ag & 2.6138 \\
\hline
\end{tabular}

From graph (1), we can determine the best enhancement for set of image using alpha - trimmed mean filter, which have less number of errors.

From below graph, we notes the uniform noise model can removed using mentioned filter with fewer errors from other noise model. 


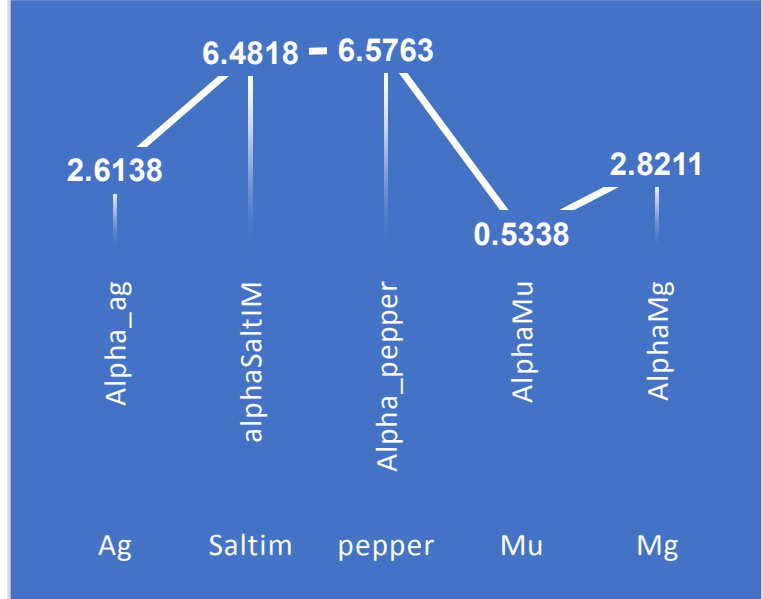

Graph (1) errors between processed images based different type of noise with alpha-trimmed filter.

On the other hand we have to apply same tool on two type of image salted image and other one have pepper noise which removed using contra harmonic filter. Then we get the results in in table (2).

Table (2) to show MSE after process image using Contra harmonic mean filter.

\begin{tabular}{|l|l||l|}
\hline ImageA & Image B & MSE \\
\hline \hline saltim & contra_saltimQ2 & 6.0321 \\
\hline \hline saltim & contra_saltimQ4 & 5.9489 \\
\hline \hline Pepper & contra_pepperQ2 & 6.2208 \\
\hline \hline Pepper & contra_pepperQ4 & 6.3578 \\
\hline
\end{tabular}

The graph (2) bellow display main difference with slated image processed with two value of $\mathrm{Q}=2$ and $\mathrm{Q}=4$ and same thing with pepper noise.

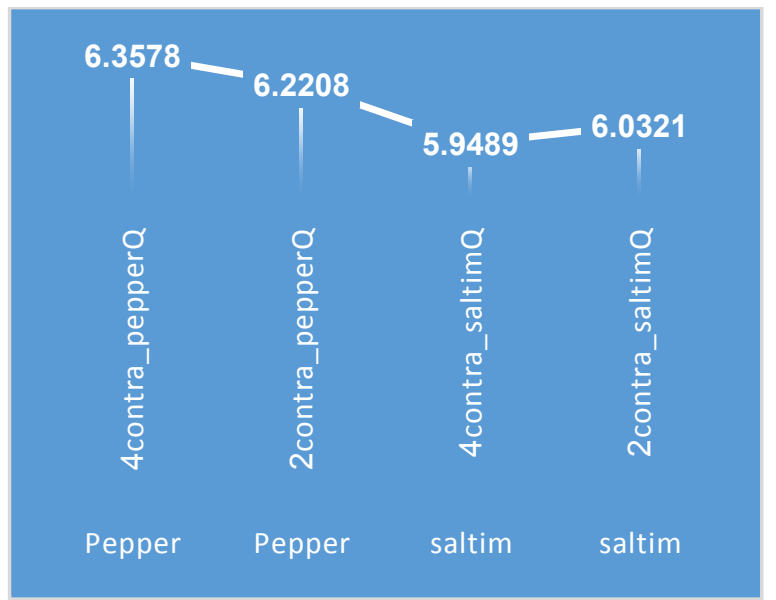

Graph (2) represent hierarchy of errors for processed image using contra-harmonic mean filter.

As show in above graph we notes the image which degraded by salt noise have number of error, moreover difference gradually increase, finally oncoming image with pepper noise and mask size $=4 \times 4$.
In another hand PSNR measure, which appear noise ratio in processed image that tool, measured by $\mathrm{dB}$ table (3) show $\mathrm{dB}$ value for each image manipulated with alphatrimmed mean filter [4].

$$
P S N R=10 \cdot \log _{10}\left(\frac{M A X_{I}^{2}}{M S E}\right)
$$

Table (3) to show PSNR after process image using alpha trimmed mean filter

\begin{tabular}{|l|l||l}
\hline Image A & Image B & PSNR \\
\hline \hline $\mathrm{Mg}$ & AlphaMg & $+27.52 \mathrm{~dB}$ \\
\hline \hline $\mathrm{Mu}$ & AlphaMu & $+27.18 \mathrm{~dB}$ \\
\hline \hline pepper & Alpha_pepper & $+39.74 \mathrm{~dB}$ \\
\hline \hline Saltim & alphaSaltIM & $+37.18 \mathrm{~dB}$ \\
\hline \hline $\mathrm{Ag}$ & Alpha_ag & $+27.49 \mathrm{~dB}$ \\
\hline
\end{tabular}

Distinctive image in above table have maximum number of PNSR, which are Alpha_pepper with +39.74 dB and that means it's best one, in another side AlphaMu image whit minimum PSNR value in addition the last mentioned image have large amount of noise as show in graph (3).

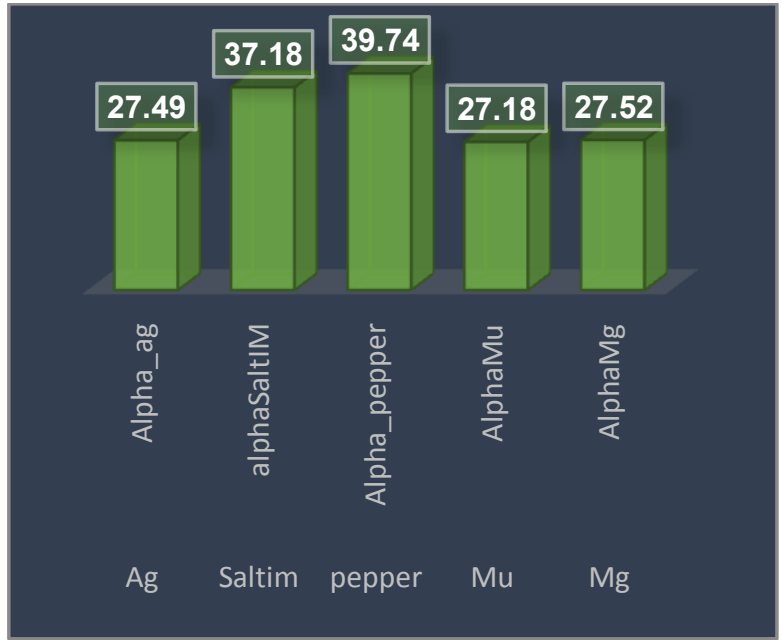

Graph (3) PSNR values of filtered image using alphatrimmed mean filter

As we nots, whenever $\mathrm{dB}$ value is high then image clear more than other images with small PSNR value.

Besides that, there is same thing with contra - harmonic mean filter as show in table (4) and graph (4).

Table (4) to show PSNR after process image using Contra harmonic mean filter

\begin{tabular}{|l||l||l|}
\hline ImageA & Image B & PSNR \\
\hline \hline Saltim & contra_saltimQ2 & $+35.49 \mathrm{~dB}$ \\
\hline \hline Saltim & contra_saltimQ4 & $+34.87 \mathrm{~dB}$ \\
\hline \hline Pepper & contra_pepperQ2 & $+39.01 \mathrm{~dB}$ \\
\hline \hline Pepper & contra_pepperQ4 & $+39.54 \mathrm{~dB}$ \\
\hline
\end{tabular}




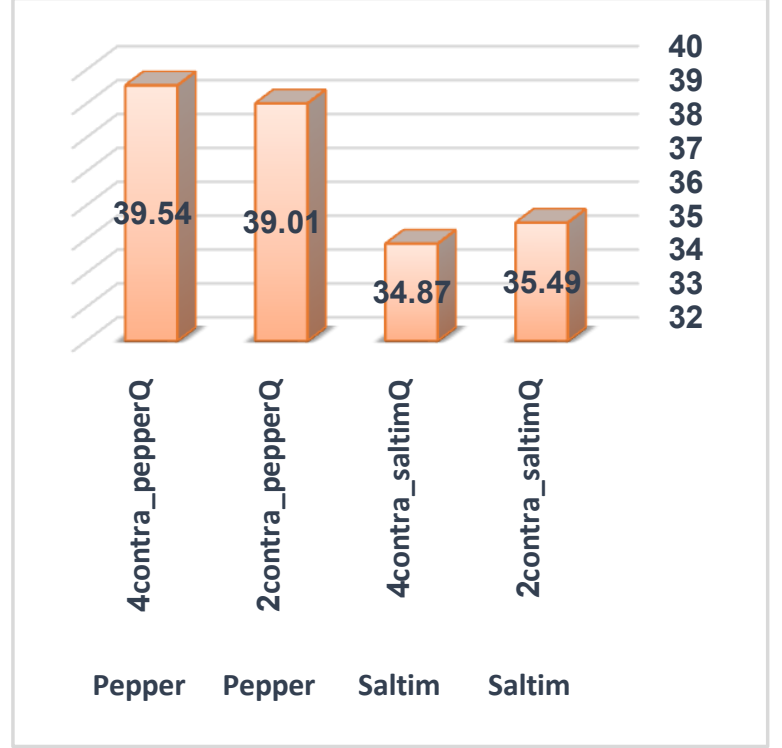

Graph (4) PSNR values of filtered image using contra harmonic mean filter

In addition to standard measurement, tools we also focus on create math model to measure how much image was been blurred after processing it [2].

Blur=Max (difference between two vertical variations of two image (input and blurred), difference between two horizontal variations of two image (input and blurred))

The idea from the blur effect is perception and estimation with a new no of reference perceptual blur metric. The value of this tool between $(0-1)$, where blur value near to 0 then there is blur in image, in the other hand if value near to 1 then image will be clear more than other images as show in table (5).

Table (5) to show difference between images based on blurring metric

\begin{tabular}{|l|l|}
\hline Processed Image & Blur Metric \\
\hline contra_saltimQ2 & 0.5784 \\
\hline contra_saltimQ4 & 0.5676 \\
\hline contra_pepperQ2 & 0.5719 \\
\hline contra_pepperQ4 & 0.5611 \\
\hline AlphaMg & 0.5787 \\
\hline \hline AlphaMu & 0.5631 \\
\hline Alpha_pepper & 0.6220 \\
\hline alphaSaltIM & 0.6198 \\
\hline Alpha_ag & 0.5866 \\
\hline
\end{tabular}

From above table, we have set of images with blur value and the following graph (5) illustrates more disparity between images.

This is graph indicate to contra_pepperQ4 image has blur more than other images, in the other side alpha-pepper with minimal blur.

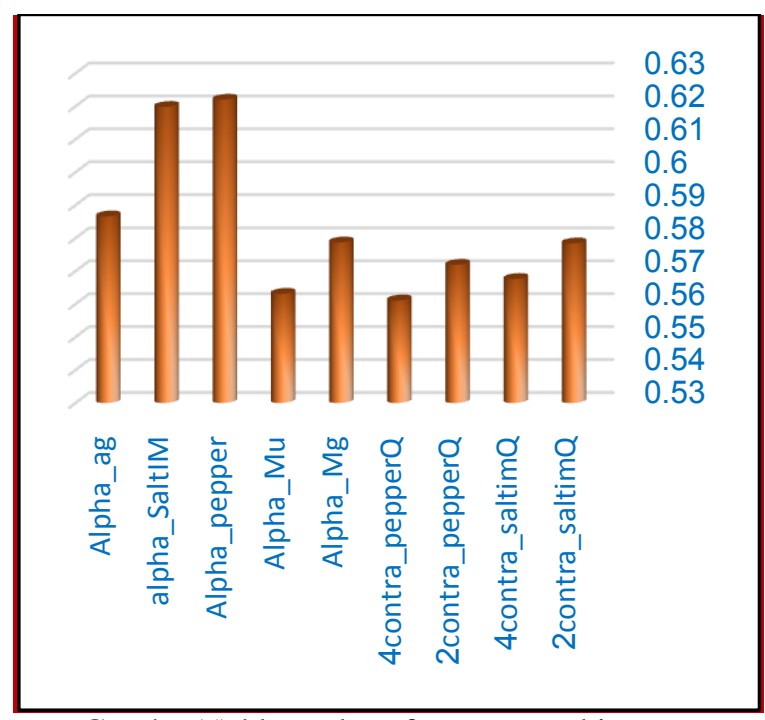

Graphs (5) blur values for processed images

As we seen from all above results Alpha-pepper image have large number of MSE value this means there is extreme difference among degraded image and processed image and that is perfect, moreover PSNR appeared as a highest value for same image, in addition blur value for Alpha-pepper image was near to 1 which means less blur from other images.

So Alpha-trimmed mean filter work on salt and pepper noise well than contra-harmonic mean filter, in addition to Gaussian and uniform noise.

\section{CONCLUSION}

From above results and analysis, we conclude both filters share with work on salt and pepper noise with perfect results for alpha-trimmed filter.

In this paper we use new technique to measure blur degree in processed image and this tool give us useful analysis to decide which one can remove noise with perfect image. Finally order statistics filters practicability, flexible and more generally because work on many noise at the same time.

\section{REFERENCES}

[1] Mahdi Shaneh, Arash Golibagh Mahyari," Image Enhancement using $\alpha$-Trimmed Mean $\varepsilon$ - Filters ", International Journal of Electrical, Computer, Energetic, Electronic and Communication Engineering volume 5, issue 11, 2011.

[2] Kanjar De and V. Masilamani, "Image Sharpness Measure for Blurred Images in Frequency Domain ", International Conference on Design and Manufacture, 2013 pp 149 - 158.

[3] R. C. Gonzalez and R. E. Woods, "Digital image processing," Third Edition, Prentice Hall.

[4] Barry C. Arnold and et.al, A First Course in Order Statistics First Edition, Society for Industrial and Applied Mathematics Philadelphia, ISBN 978-0-89871-648-1.

[5] A. Stella, Dr. Bhushan Trivedi, Implementation of Order Statistic Filters on Digital Image and OCT Image: A Comparative Study, IJMER, Vol.2, Issue.5, Sep.-Oct. 2012 pp-3143-3145. 